\title{
Bardet-Biedl Syndrome- A Rare Case Presenatation In Marathwada Region Of India
}

\author{
${ }^{1}$ Kharche Jyoti, ${ }^{2}$ Patil Vikrant, ${ }^{2}$ Yadav Pawan, ${ }^{3}$ QuaziKashifuddin, \\ ${ }^{3}$ Kothare Amit5 \\ '(Associate Professor, Department Of Medicine, MGM Medical College, Aurangabad, India) \\ 2(IIIrdYear Junior Resident, Department Of Medicine, MGM Medical College, Aurangabad, India) \\ ${ }_{3}^{3}$ IIndYear Junior Resident, Department Of Medicine, MGM Medical College, Aurangabad, India) \\ Corresponding author: *Patil Vikrant
}

\begin{abstract}
Bardet-Biedl syndrome (BBS) is a rare, genetic disorder (autosomal recessive ciliopathy) with involvement of multiple systems and wide spectrum of clinical features. Characteristic features of this disorder are cone-rod dystrophy, postaxial polydactyly, truncal obesity, kidney abnormalities and learning difficulties. It may also be associated with diminished size and decreased function of the testes in males (hypogonadism) and complex genitourinary abnormalities in females. Bardet-Biedl syndrome is inherited mostly as an autosomal recessive trait. It affects males and females equally. This syndrome is usually diagnosed in childhood based upon thorough clinical evaluation and detection of characteristic findings (e.g., visual problems due to retinal dystrophy, obesity, polydactyly). Genetic testing may assist in diagnosing the disorder in selected cases (e.g., individuals with certain BBSI and BBS10 gene mutations). The treatment of Bardet-Biedl syndrome is directed toward the specific symptoms that are apparent in each individual. Treatment may require the co-ordinated efforts of a team of specialists. We present a case of $28 y$ rs female patient with Bardet-Biedl syndrome who presented in out-patient department with episodes of loose motions, vague abdominal pain,swelling all over body and decreased urine outut. She had history of obesity, diminished vision and polydactyly. On investigations she had anemia of chronic disease, deranged renal fuction tests and retinitis pigmentosa.
\end{abstract}

Keywords: Bardet-Biedl syndrome, obesity, polydactyly, retinitis pigmentosa.

\section{Introduction}

In 1866, Laurence and Moon described a family of four siblings with retinal dystrophy, obesity, spastic paraparesis and cognitive deficit[1]. Bardet[2] and Biedl[3] later reported separately on further similarly affected individuals who in addition had post-axial polydactyly and the condition was coined Laurence-Moon-BardetBiedl syndrome. The syndrome is often divided into two entities: Laurence-Moon syndrome and Bardet-Biedl Syndrome (BBS), but there is considerable phenotypic overlap, suggesting that they may be allelic[4]. BBS is now the standard term in common usage. Mutations in at least 19 genes are known to cause Bardet-Biedl syndrome and inheritance is usually autosomal recessive ciliopathy secondary to the basal body dysfunction [5]. The frequency of the syndrome is estimated to be $1: 1,60,000_{[6]}$. Less than 15 cases have been reported from India[7]. The incidence is much higher in some populations with a high level of consanguinity or those that are geographically isolated, with disease incidence of 1 in 13,000 in the isolated populations of Newfoundland and Kuwait, 1 in 17,000 live births[8]. The characteristic combination of findings in BardetBiedl syndrome are rod cone dystrophy (93-100\%), polydactyly (58-69\%), obesity (72-88\%), learning disabilities (41-62\%), hypogenitalism in males (85-90\%) and renal anomalies (25-100\%) [9,10]. BBS is distinguished from the much rarer Laurence-Moon syndrome, in which retinal pigmentary degeneration, mental retardation, and hypogonadism occur in conjunction with progressive spastic paraparesis and distal muscle weakness, but without polydactyly[11]. Treatment depends on the symptoms present in each person. Other features not always present include diabetes mellitus, neurological, speech and language deficits, hepatic fibrosis, behavioral traits, facial dysmorphism, dental anomalies and developmental delay. Recent research has demonstrated that some individuals with the Bardet-Biedl syndrome, if diagnosed early have responded well to disease management and rehabilitation; however very limited data is available.

\section{Case report}

A 28yrs old female patient admitted to the ward with complaints of loose motions, vague abdominal pain and generalized weakness since three days and decreased urine output since two days. She underwent serial of investigations in past for complaints of progressive weight gain and swelling all over her body. She also had history of progressive loss of vision with night blindness. There was no history of a preceding viral illness, joint swelling, palpitations and breathlessness. Her BMI was $31 \mathrm{~kg} / \mathrm{m} 2$. Limb examination showed post-axial 
polydactyly in all Figure 1a: Polydactyly in lower limbs Figure 1b: Polydactyly in upper limbs Figure 2: Appearance of the patient Figure 3:Retinalpigmentary degeneration (retinitis pigmentosa) four limbs(Fig.1a, Fig.1b). She had developmental delay and was not able to learn adequately in school. No genital abnormalities were found in the patient. There was no past or family history of diabetes or thyroid disorder. On examination she was found to have pallor, obesity(Fig. 2), a low volume pulse, low BP and bilateral lower limb edema. On systemic examination, heart sounds were normal, she had hepatomegaly. On neurological examination: hypotonia, broad based gait, poor coordination, balance, dysdiadochokinesia and past pointing were present without sensory disturbance. Speech was hypernasal and slow. The IQ was 74. Hearing assessment was normal. ECG was normal. Fundus examination showed bilateral optic atrophy, rod-cone dystrophy and grossly abnormal retinal function in both the eyes, suggestive of atypical retinitis pigmentosa(Fig. 3). Complete blood counts with peripheral smear were suggestive of anemia with normocytic andhypochromic picture(Hb-6.0gm\%,TLC15060/cmm, MCV-84.9fL). renal function tests were deranged(BUN-37mg/dL, serum urea-79mg/dL, serum creatinine-3.49mg/dL, GFR-17 mL/min/m2), ESR-80mm at $1 \mathrm{hr}$. Laboratory tests including Iron studies(I$151 \mu \mathrm{g} / \mathrm{dL}$, TIBC-176 $\mathrm{g} / \mathrm{dL}$, Sat-86\%), vitamin B12 levels(>2000pg/mL), liver function(Total Bili-0.80mg/dL, SGOT-14U/L, SGPT-19U/L), serum sodium(135 mmol/L) and potassium(4.6 mmol/L), thyroid function(TSH$\left.2.5 \mu \mathrm{IU} / \mathrm{ml}, \mathrm{T}_{3}-70.95 \mathrm{ng} / \mathrm{dL}, \mathrm{T}_{4}-7.6 \mu \mathrm{g} / \mathrm{dL}\right)$, serum prolactin levels(28 ng/dL), ANA(negative), lipid profile tests(Chol-75mg/dL, TG-101mg/dL, HDL-20mg/dL) were found to be normal. bone marrow aspiration cytology study was normal. On ultrasonography there was hepatosplenomegaly with normal portal vein diameter and bilateral medical renal disease with mildly increased cortical echogenisity and preserved corticomedullary differentiation). There was no evidence of hepatic fibrosis in this case.The patient was stabilized and nephrology opinion was taken.Known risk factors for nephropathy, progressive loss of vision, obesity, I.E. recent infections, hypertension, diabetes mellitus, connective tissue disorders, inborn errors of metabolism, etc were ruled out and thus a possible familial/genetic cause was sought. Clinically she was diagnosed to be Bardet-Biedl syndrome as per modified diagnostic criteria. Genetic tests were not done due to poor socio-economic status of the patient. The patient was treated symptomatically.

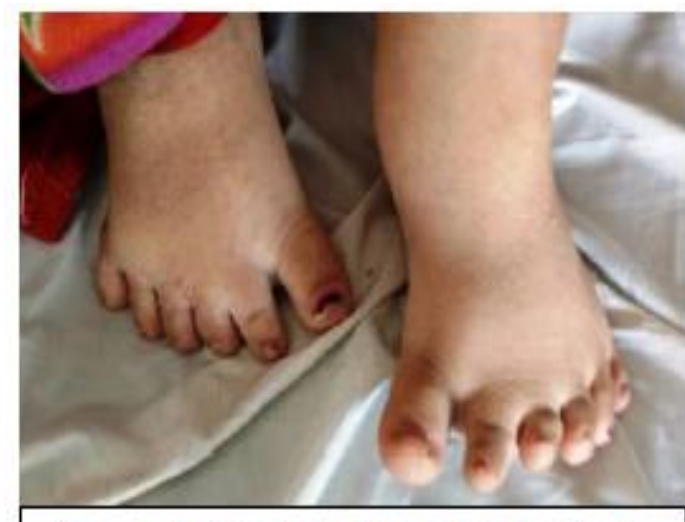

Figure 1a: Polydactyly in lower limbs

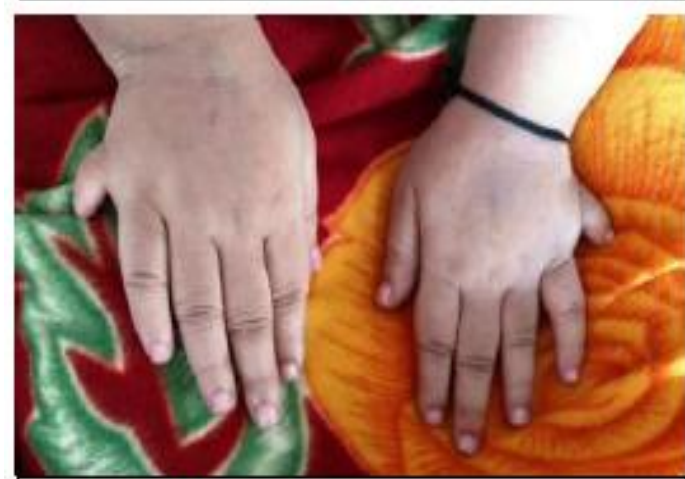

Figure 1b: Polydactyly in upper limbs

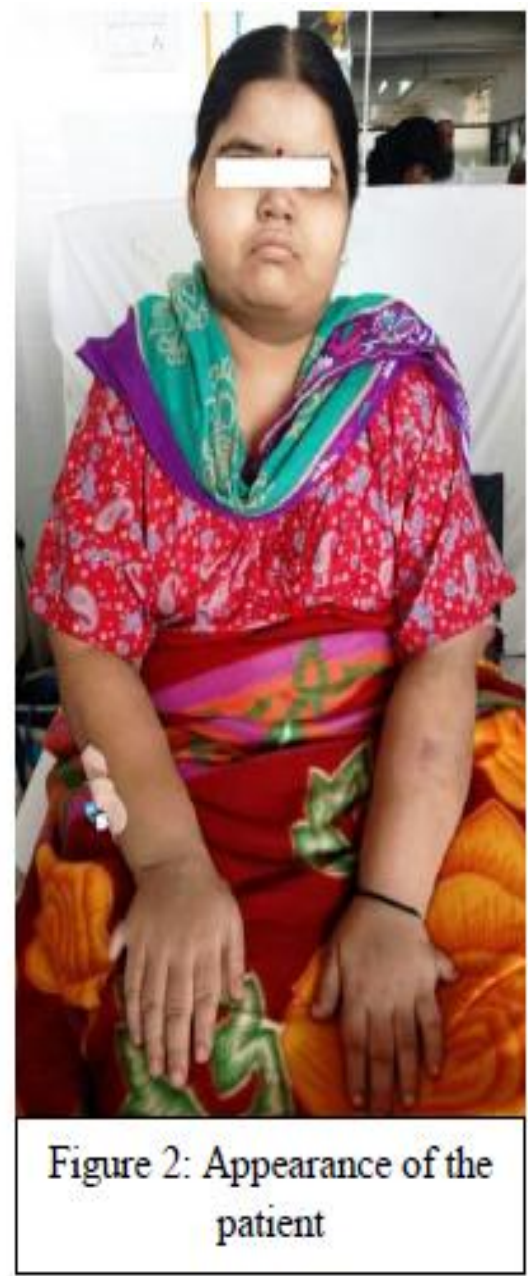




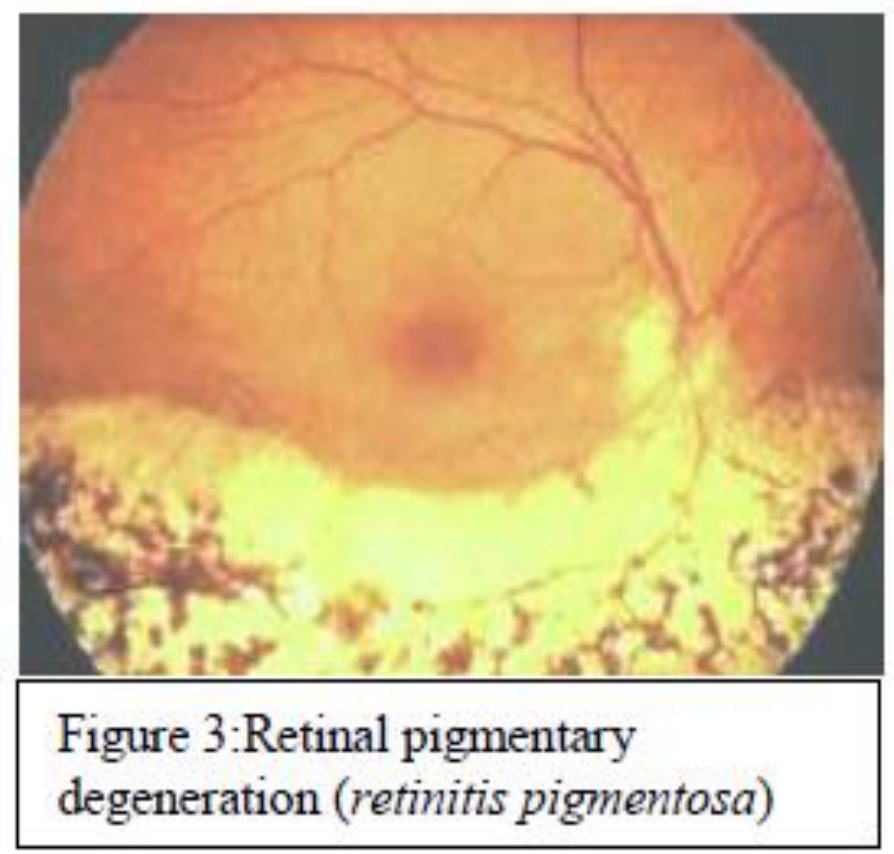

\section{Discussion}

Bardet-Biedl syndrome is named after Georges Bardet and Arthur Biedl. It is usually diagnosed in childhood based upon thorough clinical evaluation and detection of characteristic findings (e.g., visual problems due to retinal dystrophy, obesity, polydactyly). In 1999, modified diagnostic criteria were defined after a study conducted in England in 109 BBS patients[12]. Patients who had 4 primary characteristics or 3 primary and 2

secondary criteria were identified as BBS (Table 1). Genetic testing may assist in diagnosing the disorder in selected cases (e.g., individuals with certain BBS1 and BBS10 gene mutations. Investigators have determined 16 separate genes known to cause Bardet-Biedl syndrome (BBS1-BBS12), The most common defective gene associated with Bardet-Biedl syndrome is the BBS1 gene located on the long arm (q) of chromosome 11 (11q13). The gene products encoded by these BBS genes, called BBS proteins, are located in the basal body and cilia of the cell[13]. The most plausible hypothesis regarding a shared function for BBS proteins is that they assist microtubule-related transport and cellular organization processes,

in particular relating to ciliary and centrosomal activities. This hypothesis is supported by several studies using different model organisms $[14,15,16]$. Some of the phenotypes exhibited by BBS proteins, including retinal degeneration, skeletal anomalies and renal cysts/malformations bear resemblance to human diseases associated with abnormal cilia function[17]. Recently it has been demonstrated that mutations in at least 19 genes cause Bardet-Biedl syndrome and inheritance is usually autosomal recessive ciliopathy secondary to the basal body dysfunction[5]. For clinical surveillance, initial investigation and follow up of BBS patients the recommended scheme is given in table 2. The treatment of Bardet-Biedl syndrome is directed toward the specific symptoms that are apparent in each individual. Treatment may require the coordinated efforts of a team of Pediatricians, orthopedic surgeons, cardiologists, dental specialists, speech pathologists, audiologists, ophthalmologists, nephrologists, and other healthcare professionals. Individuals with Bardet-Biedl syndrome should undergo regular ophthalmologic examinations as well as periodic assessments to determine the presence of complications potentially associated with the disorder such as kidney dysfunction, diabete mellitus, liver function and high blood pressure. Early intervention is important in ensuring that children with Bardet-Biedl syndrome reach their highest potential. Genetic counselling may be of benefit for affected individuals and their families.

\section{Summary}

Bardet-Biedl syndrome is a rare, genetic disorder with variable expressivity and a wide range of clinical variability. Main clinical features are cone-rod dystrophy, postaxial polydactyly, truncal obesity, kidney abnormalities and learning difficulties. Diagnosis is based on modified diagnostic criteria with 4 primary or 3 primary and 2 secondary features, along with genetic testing in selected cases. The treatment of Bardet-Biedl syndrome is directed toward the specific symptoms and may require the coordinated efforts of a team of specialists. 


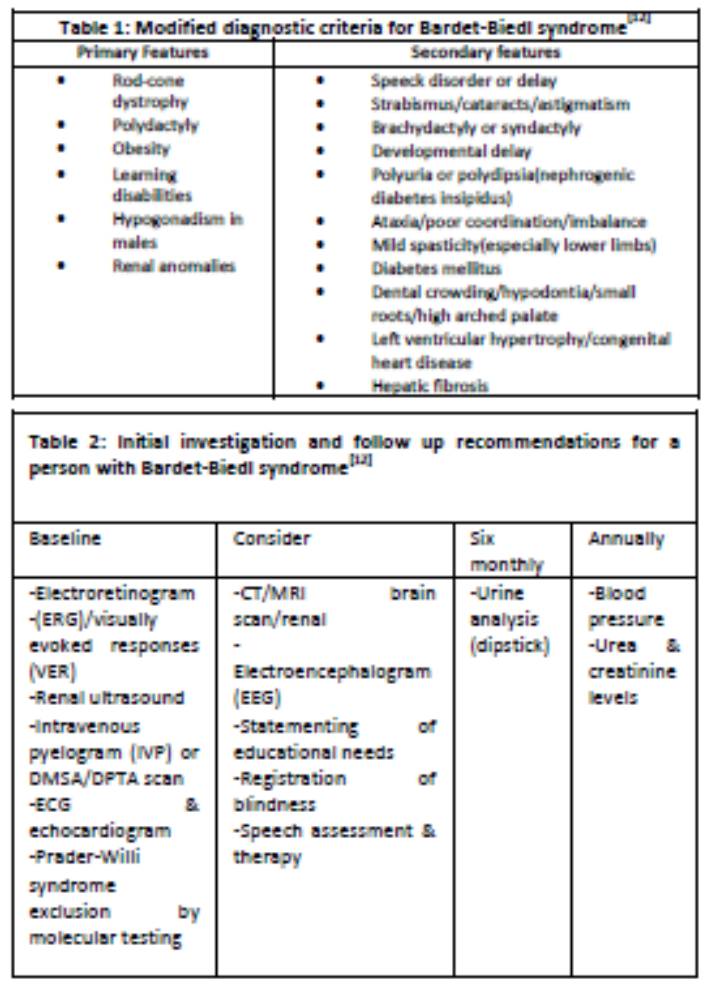

\section{References}

[1]. Laurence JZ, Moon RC. Four cases of ,retinitis pigmentosa' occurring in the same family, and accompanied by general imperfections of development. Obes Res. 1995;3:400-403.

[2]. Bardet G. On congenital obesity syndrome with polydactyly and retinitis pigmentosa (a contribution to the study of clinical forms of hypophyseal obesity) Obes Res. 1995;3:387-399.

[3]. Biedl A. A pair of siblings with adiposo-genital dystrophy. Obes Res. 1995;3:404.

[4]. Beales PL, Elcioglu N, Woolf AS, Parker D, Flinter FA. New criteria for improved diagnosis of Bardet-Biedl syndrome: results of a population survey. J Med Genet. 1999;36:437-446.

[5]. Forsythe E, Beales PL. Bardet-Biedl Syndrome. 2003 Jul 14 [Updated 2015 Apr 23]. In: Pagon RA, Adam MP, Ardinger HH, et al., editors. GeneReviews ${ }^{\circ} \quad$ [Internet]. Seattle (WA): University of Washington, Seattle; 1993-2017. Available from:https://www.ncbi.nlm.nih.gov/books/NBK1363/

[6]. Klein D, Ammann F. The syndrome of Laurence-Moon- Bardet-Biedl and allied diseases in Switzerland. Clinical, genetic and epidemiological studies. J NeurolSci 1969;9:479-513

[7]. Hooda AK, Karan SC, Bishnoi JS, Nandwani A, Sinha T. Renal transplant in a child with Bardet-Biedl syndrome: A rare cause of end-stage renal disease. Indian J Nephrol2009;19:112-4

[8]. Moore SJ, Green JS, Fan Y, Bhogal AK, Dicks E, Fernandez BA, et al. Clinical and genetic epidemiology of Bardet-Biedl syndrome in Newfoundland: A 22-year prospective, population-based, cohort study. Am J Med Genet 2005;132:352-6.

[9]. Beales PL, Elcioglu N, Woolf AS, Parker D, Flinter FA. New criteria for improved diagnosis of Bardet-Biedl syndrome: results of a population survey. J Med Genet. 1999;36: 437-46.

[10]. McLoughlin TG, Krovetz LJ, Schiebler GL. Heart disease in the Laurence-Moon- Biedl-Bardet syndrome: A review and a report of three brothers. J Pediatr. 1964;65:388-99.

[11]. Laurence JZ, Moon RC. Four cases of "retinitis pigmentosa" occurring in the same family, and accompanied by general imperfections of development. Ophthalmol Rev 1866;2:32-41.

[12]. Beales PL, Elcioglu N, Woolf AS, Parker D, Flinter FA. New criteria for improved diagnosis of Bardet-Biedl syndrome: results of a population survey. J Med Genet 1999; 36:437-446.

[13]. Ansley SJ, Badano JL, Blacque OE, Hill J, Hoskins BE, Leitch CC, Kim JC, Ross AJ, Eichers ER, Teslovich TM, Mah AK, Johnsen RC, Cavender JC, Lewis RA, Leroux MR, Beales PL, Katsanis N (October 2003). "Basal body dysfunction is a likely cause of pleiotropic BardetBiedlsyndrome".Nature425 (6958):628-33.

[14]. Ansley SJ, Badano JL, Blacque OE, Hill J, Hoskins BE, Leitch CC, et al. Basal body dysfunction is a likely cause of pleiotropic Bardet-Biedl syndrome. Nature 2003; 425:628-633.

[15]. Li JB, Gerdes JM, Haycraft CJ, Fan Y, Teslovich TM, May-Simera H, et al. Comparative genomics identifies a flagellar and basal body proteome that includes the BBS5 human disease gene. Cell 2004; 117:541-552.

[16]. Blacque OE, Reardon MJ, Li C, McCarthy J, Mahjoub MR, Ansley S, et al. Loss of C. Elegans BBS-7 and BBS-8 protein function results in cilia defects and compromised intraflagellar transport. Genes Dev 2004; 18:1630-1642.

[17]. Pazour GJ, Rosenbaum JL. Intraflagellar transport and cilia-dependent diseases. Trends Cell Biol2000;12:551-555.

* Kharche Jyoti. "Bardet-Biedl Syndrome- A Rare Case Presenatation In Marathwada Region Of India." IOSR Journal of Dental and Medical Sciences (IOSR-JDMS) 16.7 (2017): 49-52. 